\section{Aneurysm-related disseminat- ed intravascular coagulation successfully treated by endovascular repair}

\author{
Miguel Lemos Gomes, ${ }^{1,2}$ Alice Lopes, ${ }^{1,2}$ \\ Ana Parente Freixo, ${ }^{3}$ Gonçalo \\ Sobrinho, 1,2,4 Ruy Fernandes, 1,2,4 \\ Luís Mendes Pedro ${ }^{1,2,4}$ \\ ${ }^{1}$ Vascular Surgery Department, Heart \\ and Vessels Division, Hospital de Santa \\ Maria (CHLN), Lisbon; ${ }^{2}$ Lisbon \\ Academic Medical Center; ${ }^{3}$ Blood Bank \\ and Transfusion Medicine, Hospital de \\ Santa Maria (CHLN), Lisbon; ${ }^{4}$ Medical \\ School, University of Lisbon, Portugal
}

\begin{abstract}
Aortic abdominal aneurysm (AAA) is an uncommon etiology of disseminated intravascular coagulation (DIC). The authors report a case of an 81-year-old male patient who presented with hematuria, intraoral hemorrhage, melaenas and ecchymosis of the lower back and of the abdominal wall, after being medicated with etoricoxib for a back pain. During the study, an abdominal aortic aneurysm, which prolonged to the left common and internal iliac artery, was discovered. The diagnosis of AAA induced DIC was made. After endovascular aneurysm repair (EVAR), the patient's hemorrhagic manifestations disappeared and the laboratory findings normalized. In conclusion, the state-of-the-art treatment of DIC is the elimination of the underlying disease; in this case, EVAR was proven to be effective in treating the aortic aneurysm and the AAA-related DIC.
\end{abstract}

\section{Introduction}

Disseminated intravascular coagulation (DIC) is a consumptive coagulopathy caused by a great number of underlying diseases, including sepsis, trauma, malignant neoplasms, obstetric complications, severe hepatic failure, severe toxic or immunologic reactions, and vascular abnormalities. ${ }^{1}$ In an usual acute DIC secondary to infection or trauma, thrombi are formed in the circulatory system, often leading to multiple organ failure. ${ }^{1}$ Less frequently, DIC may result from a chronic gradual disease, such as vascular disorders or malignant neoplasms, and usually remains in a chronic compensated state. ${ }^{1}$ Even when clear coagulation-fibri- nolytic activation is present, the patient may show no symptoms and DIC may go unnoticed, only being diagnosed when trauma or an invasive procedure triggers sudden difficulty in achieving hemostasis, necessitating therapeutic intervention. ${ }^{2}$

Aneurysm-induced disseminated intravascular coagulopathy constitutes a rare presentation form of aortic aneurysms. The first report relating abdominal aortic aneurysm (AAA) and clinically overt DIC appeared in $1967 .{ }^{3}$ Since then, several cases were published confirming the link between these two conditions. A previous report suggested that up to $4 \%$ of aneurysms are complicated by DIC, while 39\% show subclinical elevations of fibrin degradation products. ${ }^{4}$ This occurs because there is a local activation of the coagulation cascade, occasionally resulting in the local consumption of coagulation factors and platelets with systemic hyperfibrinolysis, consequently contributing to a predominant bleeding predisposition. ${ }^{1}$

As this could provoke high mortality and morbidity, preoperative control of DIC is imperative. ${ }^{5}$ Although various agents including heparin, other anticoagulant and antifibrinolytic agents and even new oral anticoagulants (NOACs) have been used for controlling abnormal coagulopathy, the proper management of aneurysm-induced DIC is based on the treatment of the underlying disorder- surgically repairing the AAA. ${ }^{2,4,5}$ The authors describe a case where successful endovascular treatment of the aortic aneurysm corrected the DIC.

\section{Case Report}

An 81-year-old man presented at the emergency unit of another hospital with hematuria, intraoral hemorrhage, melaenas and ecchymosis of the lower back and of the abdominal wall (Figure 1). Two days before he consulted his physician due to a back pain and was medicated with etoricoxib. The past medical history included hypertension, hemorrhagic stroke, chronic gastritis (H. pylori positive), first-degree atrial-ventricular block, benign prostatic hyperplasia and vitiligo. The patient was taking aspirine (100 mg), omeprazole (20 mg), amlodipine $(10 \mathrm{mg})$ and tamsulosin $(0.4 \mathrm{mg})$ and was an ex-smoker, had a history of moderate alcohol intake and was fully independent. He had no history of surgical procedures or transfusions. The laboratory data on admission are presented in Table 1.

A diagnosis of DIC was made and he was admitted for study. Additional laboratory tests showed normal serum iron, folate
Correspondence: Miguel Lemos Gomes, Vascular Surgery Department, Heart and Vessels Division, Hospital de Santa Maria (CHLN), Lisbon, Avenida Professor Egas Moniz, 1649-035, Portugal.

Tel.: +35.1918986301.

E-mail: gomes.1.miguel@gmail.com

Key words: Aneurysm, disseminated intravascular coagulation, endovascular.

Contributions: The authors contributed equally.

Conflict of interest: The authors declare no potential conflict of interest.

Funding: None.

Received for publication: 2 June 2019. Accepted for publication: 23 October 2019.

This work is licensed under a Creative Commons Attribution-NonCommercial 4.0 International License (CC BY-NC 4.0).

(C) Copyright: the Author(s), 2019

Licensee PAGEPress, Italy

Hematology Reports 2019; 11:8189

doi:10.4081/hr.2019.8189

and B12 concentrations. During the etiologic study, a computed tomography angiography (CTA) was conducted, which revealed an abdominal aortic aneurysm, with a diameter of $71 \mathrm{~mm}$, that extended to the left common and internal iliac artery, reaching in the latter a diameter of $94 \mathrm{~mm}$ (Figure 2). The patient was then transferred to our institution and was scheduled to an endovascular treatment. The laboratory data on the day of the surgery are shown in Table 1 and a total of 5 units of packed red cells and $6 \mathrm{~g}$ of fibrinogen were given between admission and surgery. He did not have deep venous thrombosis of the lower limbs.

Under general anesthesia and after administration of 3500 units of unfractionated heparin intravenously, the left internal iliac artery was embolized with coils (Figure 3a,b). An iliac branch device (Zenith ${ }^{\circledR}$ Branch Iliac Endovascular Graft, Cook Medical, Bloomington, IN, USA) was then deployed in the right internal and external iliac arteries (Figure $3 \mathrm{c}$ and $3 \mathrm{~d}$ ) and finally, a bifurcated endovascular graft device (Zenith Flex ${ }^{\circledR}$, Cook Medical, Bloomington, IN, USA) was deployed (Figure 3e). Completion angiography demonstrated a proximal transient type I endoleak (Figure 3f). During the intervention, the patient needed 4 units of packed red blood cells, 1 pool of platelets, 3 units of fresh frozen plasma and $7 \mathrm{~g}$ of fibrinogen. In the postoperative period, the patient devel- 
oped scrotal hematoma and remained with an indwelling urinary catheter; on the $9^{\text {th }}$ postoperative day, due to elevation of the inflammatory markers, piperacillin+tazobactam was introduced empirically. A computed tomography angiography was performed on the $12^{\text {th }}$ postoperative day (Figure 4) and demonstrated no complications. The patient was discharged on the $14^{\text {th }}$ postoperative day (laboratory data - Table 1), under antibiotic therapy (amoxicillin + clavulanic acid) and maintaining the indwelling urinary catheter due to the scrotal hematoma. He was given a total of 5 units of packed red cells between the procedure and the hospital discharge.

The laboratory data at 3 months are displayed on Table 1. At 3 years, the patient remains asymptomatic, with no further hemorrhagic manifestations (Figure 5).

\section{Discussion}

After the first report of aortic aneurysm induced DIC by Fine and colleagues in $1967,{ }^{3}$ several descriptions have documented this association; nevertheless, AAArelated DIC with a bleeding tendency is a rare event $(0.5 \%$ to $4 \%))^{1,6}$ Overt DIC appears to be less frequent in patients with AAA than in those with thoracoabdominal aortic aneurysm ${ }^{1}$, due to the much larger amount of aneurysmal sac thrombus. Moreover, the diameter of AAA has been linked with the preoperative activation of coagulation and fibrinolysis. ${ }^{7}$

The DIC syndrome is a non-physiologic condition where an activation of coagulation leads to an inappropriate hemostasis with thrombosis and consumption of platelets and coagulation factors, finally determining an increased hemorrhagic risk. ${ }^{8}$ Laboratory tests reveal reduction of fibrinogen, thrombocytopenia and prolongation of INR or aPTT. Augmented fibrinol-

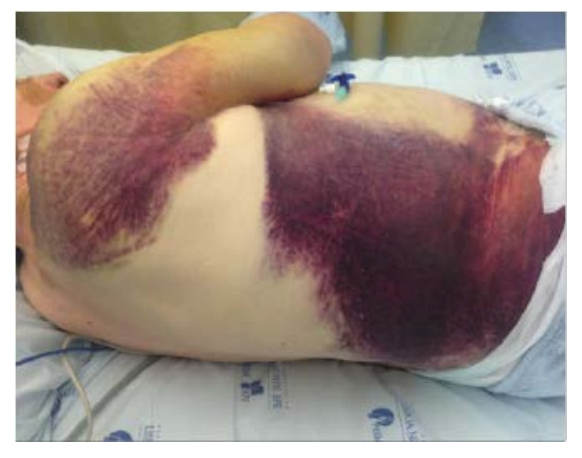

Figure 1. Ecchymosis of the dorsum and of the abdominal wall.
Table 1. Laboratory data.

\begin{tabular}{lccccc} 
& Standard & On admission Day of surgery & Discharge & 3 months \\
Hemoglobin & $13.0-17.5$ & 11.3 & 9.6 & 9.4 & 12.8 \\
WBC & $4.0-11.0$ & 5.1 & 4.28 & 6.80 & 5.57 \\
\hline Platelet & $150-450$ & 82 & 105 & 154 & 153 \\
INR & & 1.6 & 1.35 & 1.15 & 1.10 \\
\hline aPTT & & 41.6 & 25.9 & 34.5 & 35.8 \\
D-dimers & $0-25$ & 36727 & 37180 & 4090 & 9580 \\
\hline Antithrombin III & $80-120$ & 67.6 & 63 & & \\
Factor V & $50-100$ & 45 & 79 & & \\
\hline Factor VII & $70-130$ & 66 & 128 & & \\
Factor VIII & $50-150$ & 48 & 28 & & \\
\hline Fibrinogen & $200-400$ & 69 & 35 & 332 & 386 \\
BUN & $10-50$ & 44 & 42 & 35 & 27 \\
\hline Cr & $0-7-1.3$ & 0.85 & 0.9 & 1.0 & 0.49 \\
Na & $135-145$ & 143 & 143 & 145 & 142 \\
\hline K & $3.5-5.1$ & 3.7 & 4.4 & 4.3 & 3.8 \\
ALT & $0-34$ & 34 & 25.4 & & 32 \\
\hline AST & $10-49$ & 29 & 30 & & 29 \\
$\gamma$ GTP & $<73$ & 70 & 56 & & \\
\hline Total bilirubin & $<1.0$ & 0.62 & 1.2 & 1.6 & \\
LDH & $208-378$ & 208 & 524 & 668 & 292 \\
\hline CRP & $<0.5$ & & 1.9 & 14.5 & 3.62 \\
\hline
\end{tabular}

WBC, white blood count (cells); INR, international normalized ratio; aPTT, activated partial thromboplastin time; BUN, blood urea nitrogen; $\mathrm{Cr}$, creatinine; CRP, C reactive protein.

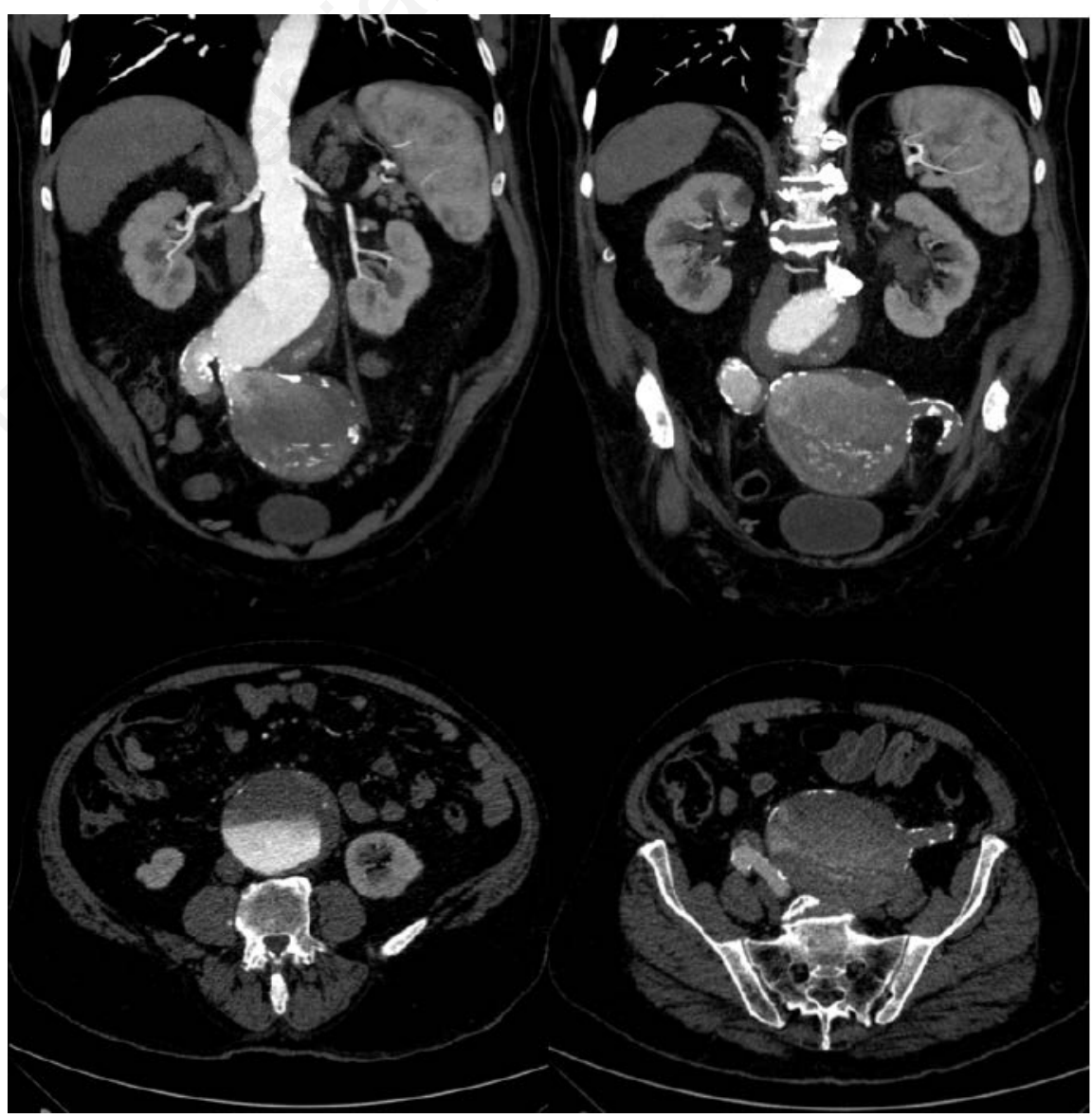

Figure 2. CTA revealing an abdominal aortic aneurysm and a left common/internal iliac aneurysm.

[Hematology Reports 2019; 11:8189] 
ysis, due to the release of tissue plasminogen activator causes increased levels of fibrin-derived products (FDPs) such as Ddimers. ${ }^{1,3,8}$ Remarkably, increased Ddimers may indicate a hypercoagulable and hyperfibrinolytic state and reflect the efficacy of treatment. ${ }^{3}$ The following criteria for DIC associated with AAA were proposed by Siebert and Natelson: presence of chronic bleeding disorder, consumptive coagulopathy, reversal of laboratory findings after aneurysm repair, and maintenance of these findings for 3 months; ${ }^{1}$ our patient fulfilled all of these criteria. We believe that the introduction of etoricoxib was the factor that triggered an otherwise chronic compensated DIC.

The mechanism of AAA-induced DIC has been extensively studied. ${ }^{3}$ Generally, it is hypothesized that turbulent flow releases thrombus from the aortic sac, exposing denuded endothelium and tissue factor, determining activation of coagulation factors, excess generation of thrombin, chronic consumption of clotting factors, and simultaneous excess plasmin generation and fibrinolysis of the clots. ${ }^{9}$

In order to attain preoperative control of DIC, allowing a safer surgery, several agents have been given; however, very few cases confirmed the effective control of DIC. Heparin is the most frequently successful drug in terms of coagulation activation and consumption regularization. ${ }^{5}$ It interrupts the vicious cycle of thrombin generation and ameliorates the DIC. ${ }^{8}$ In severe cases, with progressive bleeding resistant to heparin treatment, gabexate mesilate or nafamostat mesilate or blood components are recommended. ${ }^{3}$ Our patient had hemorrhagic manifestations (the main reason why heparin was not administrated) and the other agents are not available in our institution. That is why we only used blood components to achieve a satisfactory preoperative condition. Effective management of AAA-induced DIC by systemic administration of tranexamic acid, associated with anticoagulant agents has also been described. However, the prospect of thrombotic complications on clot dissolution has questioned the clinical efficacy and safety of tranexamic acid. ${ }^{1}$ In general for DIC, low-molecular-weight heparin (LMWH) is more appropriated than unfractionated heparin and carries a level B2 recommendation (B1 if thrombosis is present and D with severe hemorrhage), although specific recommendations are not available. ${ }^{9}$

There has been agreement that the decisive treatment of DIC is removal of the primary cause the proper management of aneurysm-induced DIC is based on the treatment of the underlying disorder. ${ }^{3}$ The most effective treatment for AAA-induced DIC is surgery, after improving the patient's general condition with blood transfusion or administration of anticoagulant. ${ }^{1}$ Presently, in suitable patients, an endovascular stent graft is an established alternative ${ }^{3}$ that excludes the aneurysm sac from the circulation, eliminating the thrombotic stimulus. ${ }^{9}$

Our patient was successfully managed by endovascular stent-graft deployment. His hemorrhagic predisposition was controlled in the early postoperative period as

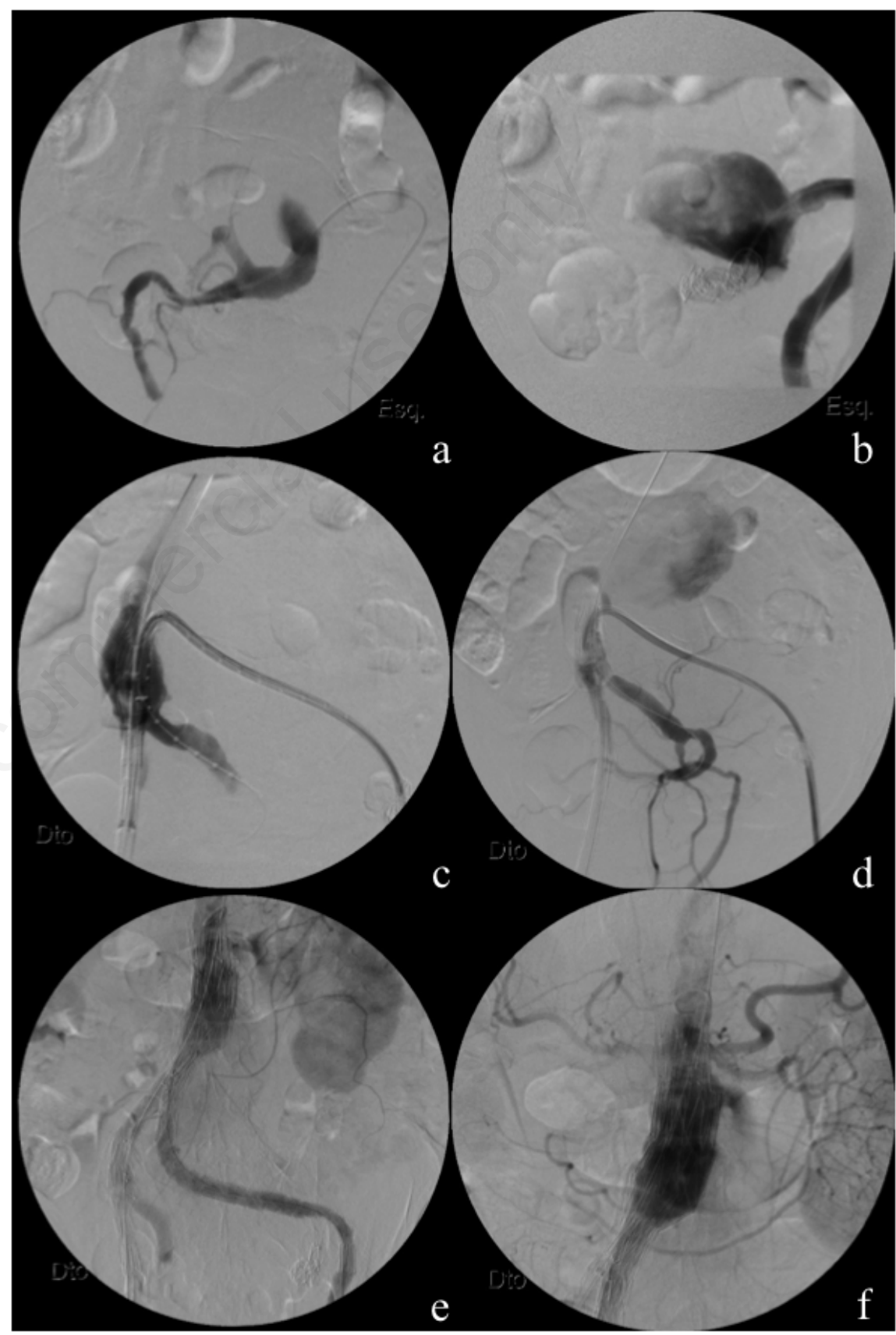

Figure 3. a) and b) Left internal iliac artery embolization; c) and d) iliac branch device being deployed in the right internal and external iliac arteries; e) control aortography; f) proximal type I endoleak. evidenced by clinical, laboratory and image data. However, follow-up of platelet counts, fibrinogen, and D-dimers levels are mandatory to ensure resolution of DIC, as all can rise as acute-phase reactants due to surgical intervention alone or decrease due to consumption while the aneurysmal sac becomes completely thrombosed (EVAR procedures). ${ }^{9}$ Control CTA is mandatory to assess the presence of endoleaks that may also be a cause of DIC. 


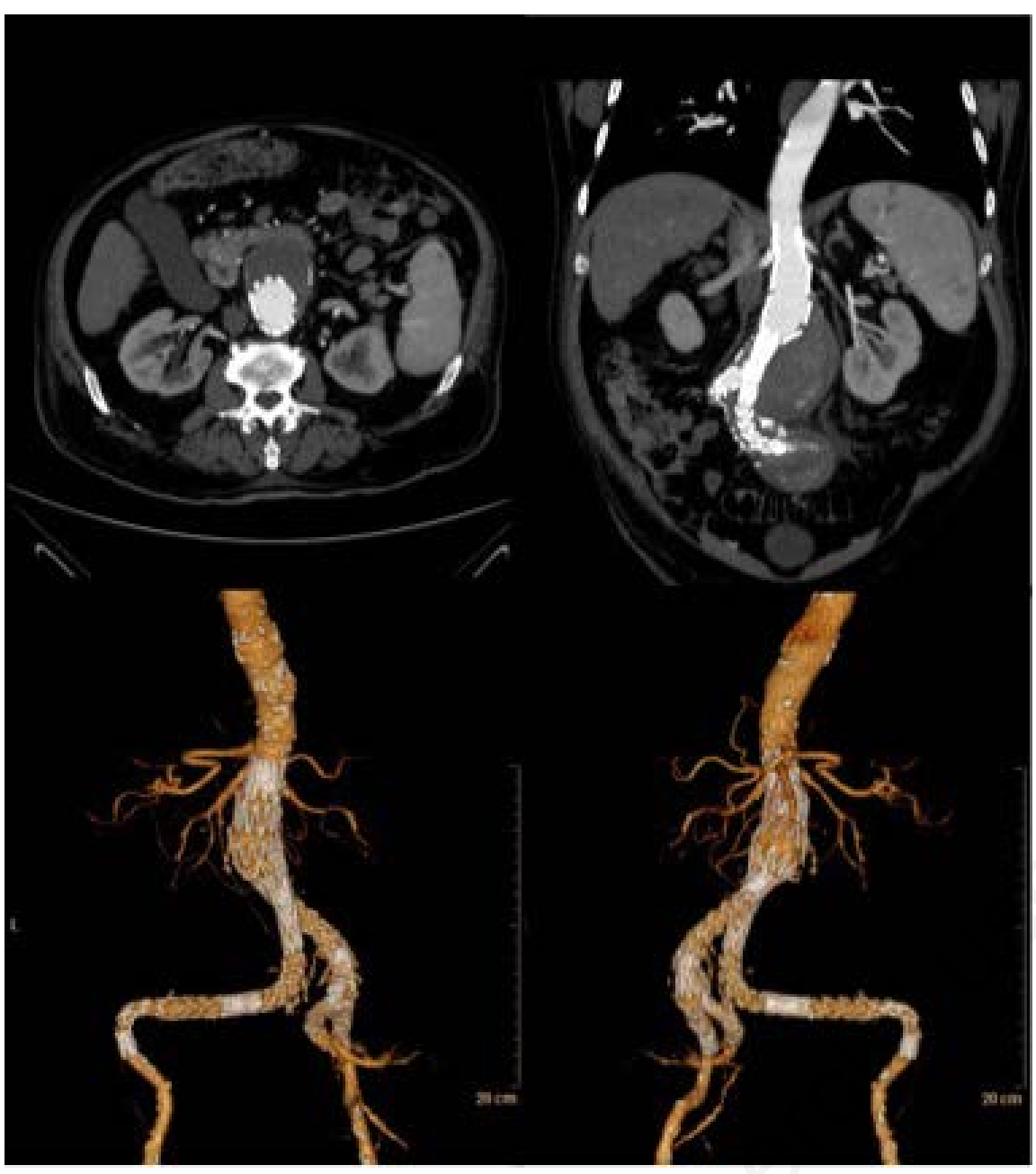

Figure 4. CTA demonstrating no endoleaks.

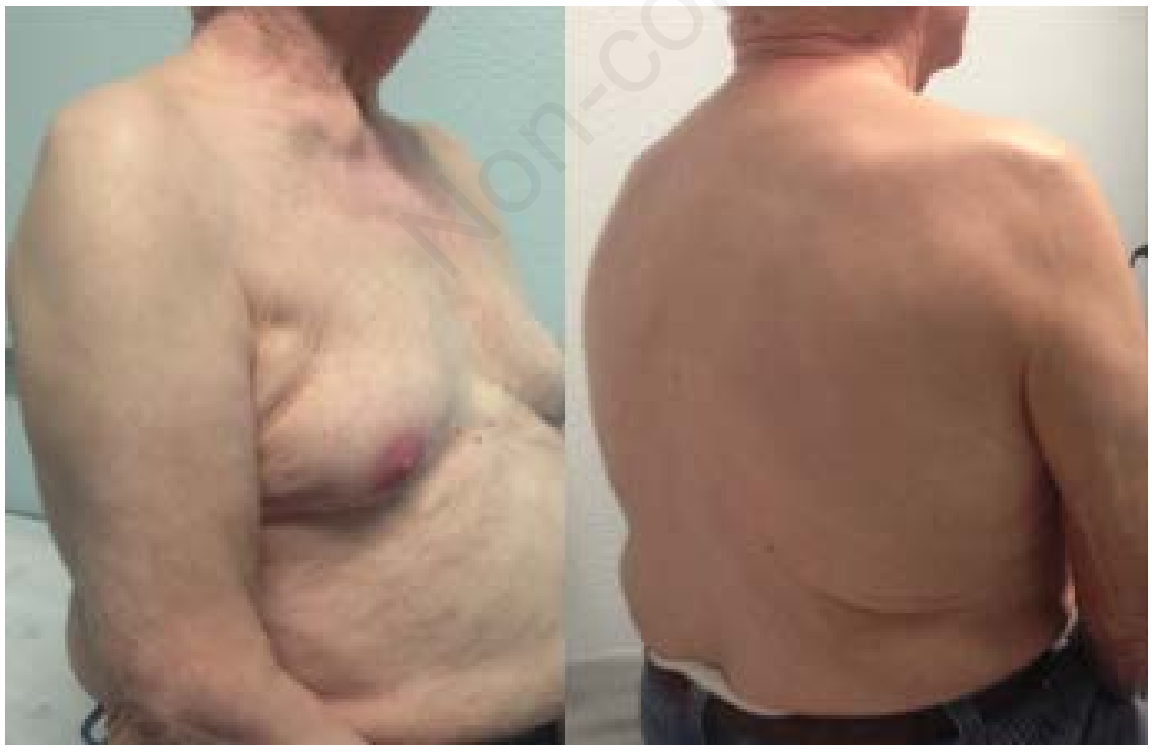

Figure 5. Total clinical recover.

\section{Conclusions}

In the patient reported, the AAA-related DIC was successfully treated by endovascular repair.

\section{References}

1. Imai $T$, Michizawa M, Shimizu H, et al. Spontaneous Intraoral Hemorrhage as Manifestation of Thoracoabdominal Aortic Aneurysm-Associated Disseminated Intravascular Coagulation: Case Report and Review. J Oral Maxillofac Surg 2010;68:195-200

2. Kadohira Y, Yamada S, Matsuura E, et al. Aortic Aneurysm-associated Disseminated Intravascular Coagulation that Responded Well to a Switch from Warfarin to Rivaroxaban. Intern Med 2017;56:2913-7

3. Yuan S, Shinfeld A, Tager S, et al. Aortic Aneurysm-Induced Disseminated Intravascular Coagulopathy: Successful Surgical Repair. Vascular 2009;17:55-9.

4. Kawano H, Hata T, Uda A, et al. Use of Rivaroxaban for the Effective Management of Disseminated Intravascular Coagulation Associated with Abdominal Aortic Aneurysm. Intern Med 2015;54:2625-8.

5. Hoshinaa K, Shigematsua K, Hosakaa A, et al. The effect of recombinant human soluble thrombomodulin on disseminated intravascular coagulation in an abdominal aortic aneurysm. Blood Coagulation Fibrinolysis 2014;25:389-91.

6. Machida H, Kobayashi M, Taguchi H. Inflammatory Abdominal Aortic Aneurysm Followed by Disseminated Intravascular Coagulation and Immune Thrombocytopenia. Intern Med 2002;41: 1032-5.

7. Jelenska M. Coagulation parameters as predictors of DIC in patients with intact aortic aneurysm. Hämostaseologie 2004;24:1626.

8. Patel A, Bell R, Hunt B et al. Disseminated intravascular coagulation after endovascular aneurysm repair: Resolution after aortic banding. J Vasc Surg 2009;49:1046-9.

9. Nienaber J, Duncan A, Oderich G, et al. Operative and nonoperative management of chronic disseminated intravascular coagulation due to persistent aortic endoleak. J Vasc Surg 2014;59:1426-9. 